\title{
Wer ssprichtı Fontanes journalistisches Gedicht »Ein Ball in Paris«?
}

\author{
Zur Diskussion um autorfaktuale Lyrik
}

\section{Einleitung}

Am 10. Dezember 1849 ist Fontane in Berlin. ${ }^{1}$ Noch bis Ende des Jahres geht er einer Beschäftigung am Krankenhaus Bethanien nach, wo ihm die pharmazeutische Ausbildung von zwei Diakonissinnen obliegt. Um eine weitere Anstellung als Apotheker bemüht er sich zu diesem Zeitpunkt bereits nicht mehr (vgl. Nürnberger 2000, 39-40). Stattdessen arbeitet er an einer literarischen Karriere, Mitte des Monats korrigiert er die Fahnen der Männer und Helden (vgl. Berbig 2010, Bd. 1, 186). Und er arbeitet an seiner journalistischen Laufbahn: Seit November schreibt er wieder einmal für ein neues Blatt, diesmal für die radikal-demokratische Dresdner Zeitung. Zu diesem Organ der sächsischen Fortschrittspartei steuert er die Korrespondenz aus Berlin bei. Nach Roland Berbig handelt es sich um Beiträge »rein politischer Natur« (Berbig 2000, 24).

In Paris jährte sich unterdessen zum ersten Mal die Wahl Louis Napoléons zum Staatspräsidenten der Zweiten Republik. ${ }^{2}$ Aus Anlass dieses Jubiläums machte man das Pariser Hôtel de Ville am 10. Dezember 1849 zum Schauplatz eines großen Balls, über den auch in der deutschen Presse berichtet wurde.

Auf welchen konkreten Wegen Fontane von dem Ereignis erfuhr, über welche Zeitungen etwa, ist nicht bekannt. Dass er von dem Ball las und dass dieser als Nachricht, Stoff, Gelegenheit - sein Interesse weckte, zeigt sich hingegen in einem Gedicht, an dem er vermutlich bereits im Dezember zu arbeiten beginnt und von dem er erste Teile, unter dem Titel »Der junge Kaiser«, im Februar 1850 in einer Tunnel-Sitzung präsentiert (»Bewertung: gut«). ${ }^{3}$ Das erste Mal publiziert wird das ausgearbeitete, schließlich 142 Verse umfassende Gedicht am 22. Mai

1 Die Theodor Fontane Chronik weist keinen Eintrag zum 10. Dezember 1849 auf; am 9. Dezember 1849 nahm Fontane an einer Tunnel-Sitzung teil (vgl. Berbig 2010, 1. Bd., 185).

$2 \mathrm{Zu}$ den historischen Hintergründen, bezogen auf Fontanes Gedicht, vgl. Peter Hasubeks gelungene Interpretation $(2001,57)$.

3 Vgl. Archiv des Literarisches Sonntagsvereins »Tunnel über der Spree«. http://allegro.ub.huberlin.de/tunnel/. »Protokoll 1850/02/24«. Datenbankabfrage im Register »Titel, Wörter aus Titeln« mit dem Suchbefehl »protokoll 1850/02/24« (29. Juli 2017).

Ә Open Access. (c) 2019 Peer Trilcke, publiziert von De Gruyter. (c) BY-NC-ND Dieses Werk ist lizenziert unter der Creative Commons Attribution-NonCommercial-NoDerivatives 3.0 Lizenz.

https://doi.org/10.1515/9783110520521-007 
1850 im Morgenblatt für gebildete Leser, nun unter dem Titel »Ein Ball in Paris« (Fontane 1850). Unter diesem Titel wird es auch - alleinstehend, ganz am Ende einen Sonderplatz in der Gedichtausgabe von 1851 finden (Fontane 1851). Die 2. Auflage der Gedichte aus dem Jahr 1875 wird den Text in die Rubrik »Bilder und Balladen« verschieben und als eine Art Untertitel die Angabe »(1849.)« ergänzen (Fontane 1875, 203). In der letzten von Fontane selbst verantworteten Auflage von 1898 lautet der Untertitel des jetzt nicht mehr in der Rubrik »Bilder und Balladen« ein-, sondern den »Gelegenheits-Gedichte[n] « zugeordneten Gedichts schließlich »(Dezember 1849)« (vgl. die Edition in Fontane 1995).

Fontane selbst hielt Ein Ball in Paris, jedenfalls 1851, für eines seiner besten Gedichte (Fontane 1995, 581 [Kommentar]). Nach dem Protokoll der Tunnel-Sitzung vom 28. April 1850, in der erneut eine Fassung gelesen wurde, verstand er es zudem als »eine Art politisches Gedicht «. ${ }^{4}$

Der folgende Aufsatz ist der Versuch, eine Antwort auf die Frage zu finden, wer diese >Art politisches` Gedicht `spricht`, wer - in der von Rüdiger Zymner vorgeschlagenen Terminologie - der >Adressant des Gedichts ist? ${ }^{5}$ Eine Antwort drängt sich dabei unmittelbar auf: Fontane, der Autor, ıspricht` dieses Gedicht nicht, er ist zwar der Autor, nicht aber der Adressant dieses Gedichts. Denn »Ein Ball in Paris « führt einen homodiegetischen `Sprecher ein (»Ich tanze nicht: im Durst nach Luft und Frische / Tret' ich seitab in eines Fensters Nische«; Fontane 1850, [485]), der als teilnehmender Beobachter den Feierlichkeiten im Pariser Hôtel de Ville beiwohnt und diese analytisch-distanziert kommentiert: der zum Beispiel den prunkvollen Aufmarsch der »schlanken Schönen « miterlebt, ihre Tänze und ihr Repräsentieren »voll fieberhafter Hast«, der darüber hinaus im Trubel den Kaiserneffen entdeckt und der all das kritisch einordnet, unter anderem indem er es mit Ereignissen aus der Revolutionszeit 1789ff. kontrastiert.

Ein solcher, reporterartig agierender Teilnehmer am Ball war Fontane nicht. Der war in Berlin und schrieb vermutlich gerade eine Korrespondenz für die Dresdner Zeitung, die am 13. Dezember - gezeichnet mit »Berlin, 10. Dez.« - unter

4 Archiv des Literarisches Sonntagsvereins »Tunnel über der Spree«. http://allegro.ub.hu-berlin. de/tunnel/. »Protokoll 1850/04/28«. Datenbankabfrage im Register »Titel, Wörter aus Titeln« mit dem Suchbefehl »protokoll 1850/04/28« (29. Juli 2017).

5 Zymner (2009, 37-47) hat auf die Problematik der Mündlichkeitsmetaphorik in der Lyrikologie, die auch und gerade den Begriff des `Sprechers` betrifft, mit Nachdruck hingewiesen. Ich teile Zymners Urteil (vgl. auch Bers/Trilcke 2017, 14-16) - und verwende den Begriff des ıSprechers`, der `spricht‘, nur in Anführungszeichen und in der Regel aus stilistischen Gründen. Wo möglich und/oder aus Gründen der Präzision erforderlich, verwende ich den von Zymner vorgeschlagenen Begriff des `Adressanten als »Markierung eines pragmatischen Ausgangspunktes des lyrischen Sprachzeichengebildes« (Zymner 2019, 26). 
dem Titel »Alles still! Das politische Leben ...» erscheint. Die Figur des an den Feierlichkeiten teilnehmenden ১Sprechers` ist selbstverständlich erfunden, ist eine lyrische Persona; Fontane selbst kann gar nicht dieser `Sprecher`sein.

Für den retrospektiv argumentierenden, über Werkausgaben, Chroniken, Bibliographien und Archivdatenbanken verfügenden Literaturwissenschaftler ist diese Aussage über den Status des Gedicht-Adressanten von geradezu zwingender Evidenz. Wenn ich im Folgenden dennoch den Versuch mache, eine Argumentation zu entwickeln, innerhalb der die gegenteilige Antwort (also: »Fontane ist der Adressant des Gedichts `Ein Ball in Paris «) einen Sinn ergibt, dann soll damit die erste Antwort keinesfalls widerlegt werden; es sei vielmehr exemplarisch darauf hingewiesen, dass die Frage nach dem Adressanten des Gedichts auf unterschiedliche Weisen beantwortet werden kann - mit gegensätzlichen Ergebnissen.

Begründen werde ich die zunächst kontraintuitiv anmutende Antwort durch eine Analyse des historischen Rezeptionskontextes, wobei ich insbesondere auf den Publikationskontext, konkret auf die Genre- und Textsortenkonventionen eingehen möchte, die die Rezeption von Fontanes Gedicht bei dessen Erstpublikation in einer Zeitschrift bedingt haben. Dahinter steht die Annahme, dass es sich bei Statuszuschreibungen wie derjenigen, auf die die Frage nach dem Adressanten eines Gedichts zielt, um sehr situationssensible Akte handelt; eine Schwierigkeit, die sie mit jenen Statuszuschreibungen teilt, die allgemein der Institution >Fiktionalitätı gelten. ${ }^{6}$

Meine Argumentation wird erst gegen Ende wieder auf Fontanes Gedicht zurückkommen. Zuvor möchte ich in zwei Schritten den systematischen und methodischen Problemhorizont umreißen, in dem sich meine Ausführungen bewegen. Der erste Schritt ruft einige grundsätzliche Schwierigkeiten und Optionen bei der Frage nach dem Adressanten des Gedichts in Erinnerung und plädiert, wie eben angedeutet, dafür, bei der Suche nach Antworten die historischen Publikationskontexte und deren Logiken zu berücksichtigen (Abschnitt 2). Im zweiten Schritt werde ich in Auseinandersetzung mit einem kontextsensiblen Forschungsbeitrag von Matías Martínez die Berücksichtigung journalistischer Publikationskontexte als ein Desiderat der lyrikologischen Diskussion identifizieren (Abschnitt 3). Welche Konsequenzen die Einbindung solcher Kontexte für die Beantwortung der Frage nach dem Adressanten eines Gedichtes haben kann, werde ich dann exemplarisch mit Blick auf Fontanes »Ein Ball in Paris« darlegen (Abschnitt 4).

6 Zur Institution Fiktionalität vgl. Köppe (2014a). Die Frage nach dem ıSprecher ` des Gedichts steht im weiteren Kontext der Diskussion um die Fiktionalität bzw. Faktualität von Texten, ich werde entsprechend im Folgenden wiederholt auf Forschungen zu diesem Feld zurückgreifen. 


\section{Systematische Fragen nach dem iSprecher des Gedichts}

Wenn es in Theorien der Lyrik um die Frage geht, wer der (so die zumeist verwendete Bezeichnung) `Sprecher eines Gedichtes sei, begegnet noch heute gelegentlich ein merkwürdiger Hiat zwischen literaturwissenschaftlicher Norm und dem, was als Lesewirklichkeit angenommen wird. So konstatiert etwa Jochen Strobel 2015 in seiner Einführung in die Gedichtanalyse zunächst: »Das Ich darf nicht mit der Autorin oder dem Autor verwechselt werden « - lässt dieser normativen Aussage jedoch sogleich die deskriptive Nebenbemerkung folgen: »auch wenn Leser/innen und Forschung gerade aufgrund der unterstellten Lebendigkeit, Unmittelbarkeit und Erlebnishaftigkeit von Gedichten immer wieder den Verfasser im Sprecher gespiegelt sehen wollten« (Strobel 2015, 219). Ähnliches lässt sich auch bei Jochen Petzold beobachten: Es sei eine »Tatsache, dass der Sprecher im Gedicht häufig mit dem realen Autor gleichgesetzt wird «, hält Petzold fest; ja man könne dies als »Beschreibung realer Rezeptionsstrategien« (Petzold 2012, 131) ansehen. In seinem theoretischen Entwurf konzipiert er das Gedicht jedoch konsequent als einen »fiktionalen Sprechakt « (Petzold 2012, 115 sowie Kap. 4.3.2.), wobei er eine klare Trennung von >realem Autor` und >primärer Sprechinstanz` ansetzt, zwischen denen allenfalls eine >Nähe bestehen könne (vgl. z. B. Petzold 2012, 143 und 120).

Folgt man der Logik dieser und vergleichbarer Aussagen, dann steht letztlich das Urteil, die Leserinnen und Leser gingen schlichtweg falsch mit Gedichten um. Nun spricht zwar nichts dagegen, einen (in der Regel offenkundigen) Unterschied zwischen der theoretisch informierten Diskursivierung von literarischen Texten auf der einen Seite und dem populären Umgang mit diesen auf der anderen zu konstatieren, also festzuhalten, dass >Laien ‘ anders lesen als Literaturwissenschaftlerinnen und Literaturwissenschaftler. Eine Theoriebildung jedoch, die zu dem Schluss kommen muss, dass die tatsächlichen Rezeptionsroutinen in weiten Teilen falsch sind, halte ich für verfehlt. Wo wir es nicht mit einem vereinzelten misreading zu tun haben, sondern mit einer (so Strobel bzw. Petzold) »immer wieder« bzw. »häufig« zu beobachtenden, das heißt mehr oder weniger konventionalisierten Praxis des Umgangs mit Literatur, da stellt sich der Lyrikologie vielmehr die Aufgabe, diese Konventionen in ihre Theoriebildung einfließen zu lassen.

Geschehen ist das unter anderem in Rüdiger Zymners klärendem Vorschlag einer Systematik. ${ }^{7}$ Unter anderem die Ausführungen von Frank Zipfel (2001,

7 Ich verzichte an dieser Stelle auf eine Diskussion der zahlreichen anderen Systematiken und Theoretisierungen des lyrischen `Sprechers`, Subjekts, Ich und dergleichen - von Hegel über Sus- 
299-304) aufgreifend, hat Zymner dargelegt, dass es in der Lyrik, wie in anderen "Spielwerken der Literatur «, sowohl möglich sei, »daß der Autor selbst ıspricht»", als auch, »daß der Autor eine Figur (oder Persona) erfindet « (Zymner 2009, 11); Zymner unterscheidet also zwischen auktorialen und personalen Gedicht-ıSprechern ‘ bzw. Adressanten: Lyrik kann, so Zymner, autorfiktional oder autorfaktual sein oder aber personafiktional oder personafaktual. ${ }^{8}$ Dass Leserinnen und Leser >immer wieder dem Sender, dem `Sprecher eines Gedichtes dessen Autorin oder Autor erkennen (und sie also als autorfiktional oder autorfaktual deuten), erweist sich im Rahmen einer solchen Systematik nicht mehr als Fehlverhalten, sondern als eine von mehreren sinnvollen Umgangsweisen mit lyrischen Texten.

Mit dieser systematischen Klärung ist allerdings nur ein erster Schritt getan. Im Anschluss stellt sich die Frage, aufgrund welcher Argumente, Kriterien, Quellen, Signale, Indizien etc. entschieden wird, welche Umgangsweise angesichts eines konkret vorliegenden Gedichtes angemessen ist.

Die von Zymner (2009, 11-12) angeführten Beispiele zu seiner Systematik liefern hier zwar erstes Anschauungsmaterial, doch keinerlei Beweisführung. Warum etwa Fontanes Gedicht »Wunsch» (»Ich wollte, daß in Sturmesnacht / Die Mutter mich zur Welt gebracht«; Fontane 1995, 340) zum Typus jener Gedichte zählt, in denen »der Autor selbst `spricht ` und dabei Erfundenes oder Erdichtetes mitteilt« (Zymner 2009, 11), wird nicht begründet. Dabei ist es doch denkbar, dass Fontane für dieses Gedicht ein Rollen-Ich, also eine Figur bzw. Persona, erfunden hat, um die Denkweise eines nach Stärke und Taten dürstenden Subjekts vorzuführen, zu inszenieren. ${ }^{9}$ Reichen der Zeitbezug, ${ }^{10}$ die Selbstcharakterisierung des Ich als `Liederschaffender`sowie die Ähnlichkeit zu von Fontane bekannten Klagen über die eher schwächliche eigene Konstitution schon aus, um sich sicher zu sein, dass es in diesem Fall der Autor selbst ist, der einen »Wunsch « äußert? Dass wir es also, in Zymners Worten, nicht mit einer Persona, sondern mit einer auktorialen Textfiguration zu tun haben?

Unabhängig von der Frage nach Fontanes Gedicht führen diese Kommentare vor allem die erhebliche Interpretationsabhängigkeit solcher Einordnungen vor Augen. Auch für die spezielle Frage, wer das Gedicht ıspricht`, gilt insofern, was

man bis Hamburger und weit darüber hinaus. Verwiesen sei stattdessen auf die Ausführungen von Zymner (2009, 10-20) sowie von Borkowski und Winko (2011).

8 Vgl. die entsprechenden Einträge in Zymners Glossar »Lyrikologische Begriffe« (Zymner 2019). 9 »Das Auge hell, im Arme Mark, / Frisch wie der Quell, wie Eichen stark«; »Im Busen lebte mir die Kraft, / Die Taten statt der Lieder schafft« (Fontane 1995, 340).

10 »nah ist der Streit, / Und ganze Männer heischt die Zeit«, schreibt Fontane in den 1840er Jahren (Fontane 1995, 340 und 640 [Kommentar]). 
Zipfel allgemein für Aussagen über den fiktionalen oder faktualen Status von Texten bemerkt hat, dass nämlich »die Ermittlung des Status eines Textes letztlich eine ebenso komplexe Interpretationstätigkeit erfordern kann wie das Verständnis des Textes als solchem« (Zipfel 2014, 120). Angesichts des Methodenpluralismus in der Literaturwissenschaft bedeutet dies auch, dass das Urteil über den ১Sprecher des Gedichts in hohem Maße abhängig ist von der jeweils gewählten Interpretationskonzeption und der im Hintergrund operierenden Literaturtheorie. Auch darin mag einer der Gründe dafür liegen, dass die Frage, wer der Adressant eines Gedichts ist, wer es also `spricht`, in der Lyrikologie notorisch umstritten ist.

Nun gäbe es einen möglichen - allerdings selbst wiederum literaturtheoretisch spezifisch justierten - Ausweg aus dieser Interpretationsabhängigkeit, und zwar die entschiedene Empirisierung der Frage im Sinne der historischen Rezeptionsanalyse (Mellmann und Willand 2013). ${ }^{11}$ Welche Zuschreibungen, so würde die Frage dann lauten, haben reale Leserinnen und Leser angesichts konkreter Gedichte vorgenommen? So groß das Potenzial einer solchen Wendung ist, so beschränkt ist zugleich ihre Reichweite. Ließen sich für gegenwärtige Texte noch empirische Befragungen durchführen, wäre man für historische Texte auf überlieferte Rezeptionszeugnisse angewiesen. Diese aber, ohnehin vergleichsweise selten und bei Weitem nicht repräsentativ, liefern nur im Ausnahmefall Hinweise auch auf die infrage stehende Adressanten-Statuszuschreibung. ${ }^{12}$ Damit aber ist eine Beantwortung der Frage in vielen Fällen unmöglich.

Eine Lyrikologie, die bei Erstellung ihrer systematischen Modelle die Rezeptionswirklichkeit nicht ausblenden möchte, kommt insofern angesichts der dürftigen Quellenlage nicht daran vorbei, mit voraussetzungsreichen Interpretationen zu argumentieren. Von dem Programm der historischen Rezeptionsanalyse übernehmen kann sie die Forderung nach einer forcierten Historisierung. Bei der Beantwortung der Frage, wer das Gedicht ıspricht`, wären in diesem Sinne zumindest die historischen Rahmenbedingungen, in denen sich die individuellen Rezeptionsakte vollzogen haben, möglichst umfangreich zu berücksichtigen. Ein solcher historischer Rezeptionskontext umfasst, neben der allgemein sepistemischen Situation` (Lutz Danneberg), unter anderem Aspekte wie das (`häufig` populäre) Wissen um generische Konventionen, vor allem in Hinblick auf typische `Sprecher<-Figurationen, um die Kommunikationslogik einzelner Publikationstypen, -formate und -organe oder um den kontextsensiblen Signalcharakter

11 Wobei auch die Analyse von Rezeptionszeugnissen auf interpretativen Akten beruht (Mellmann und Willand 2013, 281).

12 Vgl. zu diesem Problem - in Hinblick auf die Institution >Fiktionalität ‘ - auch die allgemeinen Reflektionen bei Tilmann Köppe (2014b, 421-422). 
bestimmter Textphänomene hinsichtlich der Institution >Fiktionalitätı. Der historische Rezeptionskontext ist dabei stets ein unvollständiges, weil selektives literaturwissenschaftliches Konstrukt, das entsprechend diskutabel, korrigierund falsifizierbar ist - aber es ist aus meiner Sicht ein notwendiges Konstrukt. ${ }^{13}$

Mit den zurückliegenden Ausführungen sollte ein erster Problemhorizont aufgespannt werden, in dem sich die sowohl systematische als auch historische Frage, wer der Adressant eines Gedichts sei, bewegt. In Auseinandersetzung mit einem konkreten Forschungsbeitrag soll im folgenden Schritt jenen methodischen Aspekten weiter nachgegangen werden, die bereits im Zusammenhang mit Zymners Einordnung von Fontanes »Wunsch« eine Rolle spielten. Im Zuge dessen möchte ich auch darauf aufmerksam machen, dass in der systematischen Diskussion über die Möglichkeit einer Identifikation von Adressant und Autor zwar immer wieder der Autor als biographisches Subjekt ins Spiel gebracht wird, selten aber als - wie ich es nennen werde - publizistisches Subjekt.

\section{Biographische und publizistische Gedicht-ıSprecher}

Auch wenn die Position, es sei keinesfalls die Autorin oder der Autor, die ein Gedicht ssprechen ', sich weiterhin erheblicher Beliebtheit erfreut, gibt es durchaus Beiträge, in denen begründet dafür argumentiert wurde, den `Sprecher des Gedichts mit der Autorin, dem Autor gleichzusetzen. Wie dabei vorgegangen wurde, sei exemplarisch an Matías Martínez’ »Verteidigung« des Begriffs >lyrisches Ich rekonstruiert.

Eines der Gedichte, die Martínez in seinem Aufsatz diskutiert, ist Goethes „Vom Berg in die See« (»Wenn ich liebe Lili dich nicht liebe«) (Martínez 2002, 383-386). Die zentrale argumentative Funktion übernimmt der Publikationskontext von Goethes Gedicht, das dieser zunächst im Sommer 1775 in sein Tagebuch schrieb, 1789 dann in seinen Schriften veröffentlichte und Jahrzehnte später noch einmal in Dichtung und Wahrheit abdrucken ließ. Damit habe Goethe »einen weitgehend identischen Text in zwei unterschiedliche Kontexte gestellt, einerseits in

13 Im Grunde teile ich Marcus Willands Plädoyer, dass für »eine adäquate Historisierung lediglich faktische Rezeptionszeugnisse realer Leser « $(2014,44)$ berücksichtig werden sollten, sehe aber zum einen das Problem der Überhöhung einzelner Zeugnisse (mithin das Problem ihrer Repräsentativität), zum anderen das allgemeine Problem des Fehlens von Zeugnissen überhaupt. 
einen biographischen (in Reisetagebuch und Autobiographie), andererseits in einen literarischen (im Gedichtband)« (Martínez 2002, 383).

Daraus resultierten, so Martínez, unterschiedliche Voraussetzungen, die auch unterschiedliche Umgangsweisen mit dem Text als angemessen erscheinen lassen. Während das Gedicht beim Abdruck im Tagebuch und in der Autobiographie »in einem praktischen Kommunikationszusammenhang mit einem bestimmten Sender [also Adressanten, P. T.], Goethe, und einer bestimmten Adressatin, Lili Schönemann «, stehe, gehe dieser »reale[ ] Situationsbezug " und also auch die »Zuordnung zur empirischen Person Goethe als Sprecher des Textes« (Martínez 2002, 385) beim Abdruck im Gedichtband verloren. Im ersten Fall ıspricht` also Goethe das Gedicht, im zweiten nicht.

Martínez' Identifikation der »Person Goethe« als »Sprecher des Textes« basiert auf zwei Beobachtungen: Zunächst identifiziert er, als Teil des historischen Rezeptionskontextes, spezifische Publikationsformate (Reisetagebuch, Autobiographie) des Gedichts, wobei diese Formate in der Regel faktuale Kommunikation indizieren. Zudem kann er konstatieren, dass die im Gedicht getätigte Aussage sehr gut zu der »Person « Goethe passt. ${ }^{14}$ - Auch ich halte es angesichts dieser Beobachtungen für kontraintuitiv, noch eine Instanz wie ein `Textsubjekt oder einen simpliziten Autor anzusetzen. Martínez' Argumentation zu Goethes Gedicht ist aus meiner Sicht plausibel.

Nicht vergleichbar überzeugend, weil unvollständig ist demgegenüber die Argumentation in Zusammenhang mit Bertolt Brechts Gedicht »Aus allem etwas machen«. Martínez hält hier zwei Punkte fest. Erstens hat auch dieses Gedicht eine »reale Referenz«, allerdings nicht, wie bei Goethe, »auf eine private, sondern auf eine allgemein bekannte politische Situation« (Martínez 2002, 387). Zweitens konstituieren die Verse, anders als diejenigen Goethes, »weder ein personales noch ein räumliches deiktisches System«; es werde »keine konkrete Sprechsituation evozier[t]« (Martínez 2002, 387). Diese zwei Punkte lassen Martínez zu dem Schluss kommen, dass der »Gedichttext [...] keiner realen Kommunikationssituation [angehört] « (Martínez 2002, 388). Brechts Gedicht stelle insofern einen zu Goethes Versen »möglichst entgegengesetzten Fall« dar (Martínez 2002, 386); eine Identifikation des Adressanten mit der Person Brecht nimmt Martínez entsprechend an keiner Stelle vor.

Bemerkenswert an diesem Kommentar zu Brechts Versen ist, was er im Vergleich mit dem Kommentar zu Goethes Gedicht nicht thematisiert, das ist zum einen die spezifische Logik des Publikationsorgans, in dem das Gedicht ver-

14 Unter anderem aufgrund des im Kontext Ausgeführten, aus dem hervorgeht, dass Goethe Lili in der Tat liebt. 
öffentlicht wurde, zum anderen die `Näheく oder auch >Passung` zwischen der im Gedicht getätigten Aussage und dem, was wir über den Autor, die empirische Person Brecht, wissen. Gerade also die beiden Aspekte, die bei der Diskussion der Goethe-Verse entscheidend waren, spielen beim Kommentar zum BrechtGedicht keine Rolle mehr. Allerdings ließe sich auch angesichts von Brechts »Aus allem etwas machen « überlegen, ob nicht eine Identifikation der Person Brecht als Gedicht-Adressant nahe liegt. Denn auch dieser Text wurde (was Martínez kurz erwähnt) nicht in einem Gedichtband publiziert, sondern in einem anderen Kontext, in diesem Fall in einer Zeitschrift: 1949 im ersten Jahrgang von Sinn und Form (Brecht 1949). Zwar indiziert dieser Kontext, anders als eine Autobiographie, keineswegs bereits konventionell Faktualität, doch versammelten die Sinn und Form-Hefte in ihrem "multi-discursive space [...] texts from a range of literary and non-literary genres « (Parker und Philpotts 2009, 279); in der Ausgabe, in der Brechts Gedicht erschien beispielsweise auch Briefe und Essays. Es war also keineswegs eindeutig, was für einen Status ein Text in Sinn und Form hatte. Hinzu kommt, dass Brecht 1949, soeben angekommen in der SBZ bzw. DDR, ein Interesse daran hatte, den Aufbau der DDR zu unterstützen: ${ }^{15}$ Ein Gedicht wie »Aus allem etwas machen «, das anhand einer Anekdote ${ }^{16}$ aus dem maoistischen China veranschaulicht, wie aus einer Situation des Mangels und der ideologischen Anfeindung heraus nützliches Handeln hervorgeht, passt da sehr gut.

Denkbar wäre es also, dass es sich bei dem in Sinn und Form abgedruckten Gedicht um eines, wie Brecht in den 1920er Jahren eingefordert hatte, mit »Gebrauchswert (Brecht 1992, 191) handelt. Um ein Gedicht, das möglichst nah an der »ursprünglichen Geste der Mitteilung eines Gedankens« (Brecht 1992, 191) operiert. Dass Brechts Gedicht, wie Martínez schlussfolgert, »keiner realen Kommunikationssituation« angehöre, wäre damit so zwingend nicht, ja dieses Urteil mutet sogar recht kontrainiutiv an. Sicher, dies wäre keine Kommunikationssituation wie diejenige, in der Goethe sich Ich-sagend an seine liebe Lili wendet. Es würde sich hier vielmehr der Adressant Brecht, gewillt, am Aufbau der DDR mitzuwirken und zu diesem Zweck eine Geschichte vom ıweisen Handeln Maos erzählend, an den Adressaten `Volkı richten. ${ }^{17}$ Dieser Brecht `spräche` dabei nicht als ein biographisches, sondern als eine Art publizistisches Subjekt.

15 Ende 1948 waren das »Aufbaulied der F. D.J.« und das »Zukunftslied« entstanden; in einem Brief vom 1. Juli 1949 an Paul Huchel, dem Redakteur von Sinn und Form, lobt Brecht die Zeitschrift als "geistreiche und planmäßige Kontribution zum Aufbau und Umbau« (Brecht 1998, 540).

16 Die er kurz zuvor in der Zeitung gelesen hatte (vgl. Brecht 1993, 438 [Kommentar]).

17 »Nach der Rückkehr nach Deutschland suchte Brecht zunächst wieder den Adressaten, den die Exillyrik nicht haben konnte, und zwar das `Volkı, dessen `Weisheit « er für den Wiederaufbau und den Aufbau des Sozialismus für unverzichtbar hielt« (Knopf 2000, 242). 
Anders als in historischen Studien haben diese publizistischen Subjekte, Situationen und Kontexte in der systematischen Diskussion der Lyrikologie bisher nur am Rande eine Rolle gespielt. Wo es darum ging, die Möglichkeit der Identifikation von Gedicht-Adressant und Autorin in Erwägung zu ziehen, stand meist ein sich selbst bespiegelndes oder seine Emotionen ausdrückendes Subjekt im Mittelpunkt: So etwa in Carolin Fischers Arbeit zum spoetischen Paktı, die sich schwerpunktmäßig der Liebeslyrik widmet (Fischer 2007); oder bei Dieter Burdorf, der den »kategorialen Unterschied zwischen dem empirischen Urheber (der Autorin oder dem Autor) und dem sprachlich konstituierten Ich eines Gedichts« mit Blick auf das Gedicht als »biographisches Dokument« diskutiert. Und auch in diesem Fall gilt die Aufmerksamkeit »Goethes Liebesgedichten« (Burdorf 2015, 188).

Angesichts dieser und vergleichbarer Tendenzen der Lyrikologie ließe sich eine Entpolitisierung der Frage nach dem Adressanten des Gedichts konstatieren, jedenfalls dann, wenn man von einem Verständnis des Politischen als Gegensatz zu jener (allein biographischen, fühlenden, liebenden, vor allem aber selbstbezüglichen) Privatheit ausgeht, die meist im Zentrum steht, wenn die lyrikologische Diskussion eine Identifikation von Adressant und Autorin bzw. Autor erwägt. Ausgeblendet werden damit insbesondere all jene lyrischen `Zweckformen` (Friedrich Sengle), in denen die Autorin oder der Autor, auch ohne sich selbst als biographisches Subjekt ins Spiel zu bringen, eine Meinung oder eine Perspektive auf die Welt mitteilt, die seine, ihre eigene ist.

$\mathrm{Zu}$ einfach wäre es allerdings, in solchen Fällen ohne Weiteres von einer Identität von Autorin oder Autor auf der einen, dem Adressant des Gedichts auf der anderen Seite auszugehen. Stattdessen sollte die Lyrikologie diese Fälle ergebnisoffen in ihre systematische Reflexion integrieren. Dafür aber bedarf es der abwägenden, im skizzierten Sinne rezeptionssensiblen Analyse von Einzelbeispielen, die begründete Vorschläge für Statuszuschreibungen unterbreitet. Was ich oben als >publizistisches Subjekt ‘ bezeichnet habe, könnte hier als eine Art historischer Suchbefehl fungieren: Wo in der Lyrikgeschichte begegnen Gedicht-Adressanten, die auf den ersten Blick weder (in Zymners Terminologie) als eine von der Autorin, dem Autor erfundene Figur oder Persona noch als eine dezidiert biographischauktoriale Textfiguration begriffen werden können, sondern eher so funktionieren wie die `Sprecher`, die Kommunikationsinstanzen, die Adressanten in publizistischen bzw. journalistischen Texten, etwa im Kommentar oder der Glosse, im Bericht oder der Meldung, im Porträt oder der Reportage?

Die zurückliegenden Ausführungen haben den systematischen Stellenwert der eingangs aufgeworfenen Frage nach dem `Sprecher« von Fontanes »Ein Ball in Paris « diskutiert. Dabei ist »Ein Ball in Paris«, auch darauf wurde einleitend hingewiesen, kein eindeutiger Fall eines Gedichts mit publizistisch-auktorialer Textfiguration, wie dies etwa ein Exemplar dessen wäre, was gelegentlich >Leit- 
gedicht genannt wird. Stattdessen zeigen sich anhand von "Ein Ball in Paris« auch die Schwierigkeiten bei der Ermittlung von Statuszuschreibungen anhand historischer Rezeptionskontexte.

\section{Ein Ball in Paris im historischen Rezeptionskontext}

Eine Argumentation, nach der Fontane der Adressant von »Ein Ball in Paris« ist, wird erst dann überhaupt sinnvoll, wenn das entscheidende Argument entkräftet werden kann, das gegen diese Aussage spricht: dass Fontane gar nicht der Adressant, der 'Sprecher` sein kann, weil er an jenem Tag, an dem der `Sprecher des Gedichts in Paris einen Ball besucht, in Berlin war.

Der zeitgenössische Leser des im Morgenblatt abgedruckten Gedichts konnte dies jedoch nicht wissen, sofern er nicht gerade mit Fontane persönlich bekannt war. Und der Abdruck im Morgenblatt liefert keine Hinweise, die gegen eine Anwesenheit des Autors in Paris sprechen. Zudem hatte der regelmäßige Zeitungs- und Zeitschriftenleser vermutlich bereits von dem Ball gehört. Auch das Morgenblatt hatte in der Neujahrsausgabe unter den »Korrespondenz-Nachrichten « über das Ereignis informiert. Unter der Zwischenüberschrift »Der Ball im Rathaus« gab dort ein Autor mit dem Kürzel »Dg.« (das ist wohl Georg Bernhard Depping) einen Überblick über die Feierlichkeiten am 10. Dezember 1849 (Dg. 1850). Weder ein zeitgenössisches Wissen über den Autor, noch andere, unmittelbar das Gedicht flankierende Angaben machen also einen `Sprecher Fontane unwahrscheinlich.

Damit ist aber nur das erste, grundlegende Gegenargument zurückgewiesen. Im Folgenden möchte ich zwei weitere Gegenargumente diskutieren, die sich auf die Darstellungsweise des Gedichtes beziehen. Dabei geht es darum, zwei Textphänomene zu analysieren, die in der Forschung als starke Signale für eine Fiktionalität der Darstellung gehandelt werden. Würden diese Textphänomene auch im vorliegenden Gedicht eine derartige Signalwirkung erfüllen, dann läge es nahe, trotz der Referenz auf ein verbürgt faktuales Geschehen, von einer erfundenen 'Sprecher`-Figur, einer fiktiven lyrischen Persona also, auszugehen, ${ }^{18}$ die über bestimmte Darstellungsmöglichkeiten verfügt, die man einem realen, mit dem Autor identischen Adressanten nicht zugestehen würde.

18 In Zymners Nomenklatur läge dann der Fall eines persona-faktualen Gedichts vor (2009, vor allem 11-16). 


\title{
4.1 Der Status von Fiktionssignalen im journalistischen Kontext um 1850
}

Das erste dieser beiden Textphänomene ist die Simultaneität der Darstellung. Fontanes Gedicht beginnt so (und setzt diese gleichzeitige Geschehenspräsentation auch bis zu seinem Ende fort:)

\author{
Paris hat Ball: hin durch der Gassen Enge \\ Braust rasselnd der Carossen bunte Menge; \\ Die Quais entlang, entlang die Tuilerien, \\ Ein rastlos Jagen und Vorüberfliehn. \\ Halloh! die Peitsche knallt, die Rosse dampfen, \\ Schon dröhnt »la Grêve« von ihrer Hufe Stampfen, \\ Und jetzt ein kurzes »Halt! « - Hell glänzt das Ziel, \\ Der prächt'ge Ballsaal des Hôtel de Ville. (Fontane 1850, [485])
}

Die Verse sind im Präsens verfasst, deiktische Ausdrücke (»Schon«, »jetzt«) identifizieren den Moment der Darstellung mit dem (historischen) Moment des sich gerade vollziehenden Ereignisses. Im Sinne der von Klaus W. Hempfer vorgeschlagenen Definition ist der Text insofern prototypisch lyrisch: Die »besprochene Situation«, in diesem Fall das Ball-Geschehen, konstituiert sich »gleichzeitig» zum »Sprechakt« und »auf der Grundlage einer identischen Deixis« (Hempfer 2014, 34). Gerade aber eine solche »Simultaneität bzw. Koinzidenz von Sprechsituation und besprochener Situation« (Hempfer 2014, 34) stellt für Hempfer eine »Performativitätsfiktion « dar; sie muss mithin als ein starkes Fiktionalitätssignal gewertet werden, ${ }^{19}$ wobei die 'Fiktionalität darauf basiert, dass die Simultaneitätsrelation eine textinterne, inszenierte ist, die nicht der Kommunikationssituation zwischen Produzent und Rezipient entspricht« (Hempfer 2014, 34; meine Hervorhebungen, P. T.). Der `Sprecher von »Ein Ball in Paris « ist in diesem Sinne notwendig eine fingierte Instanz, denn derjenige, der da spricht, wird als jemand vorgestellt, der dies genau in jenem Moment tut, in dem sich der Ball ereignet. Das aber sei nur in der fiktionalen >Inszenierung`möglich. Hätte Hempfer Recht, dann würde der Text also von Beginn an signalisieren, dass hier ein fingierter >Sprecher« das Wort ergreift.

Allerdings zeigt »Ein Ball in Paris« - auch aufgrund seines journalistischen Charakters und Anliegens - die Grenzen von Hempfers Konzept der >Performativi-

19 In seinem Überblick zu Fiktionssignalen referiert Zipfel mit Blick auf die ıSituierungen des Erzählakts` eine ähnliche Position von Dorrit Cohn; auch diese »sieht im simultanen Erzählen, das heißt im Erzählen im Präsens, eine fiktionsspezifische Erzählform « (Zipfel 2014, 114). 
tätsfiktion ‘ auf. ${ }^{20}$ Denn im journalistischen Kontext, in dem das Gedicht erscheint, signalisiert die simultane Darstellung keineswegs notwendig Fiktionalität - was im Übrigen noch heute gilt, denkt man an szenisch angelegte Reportagen, bei denen keine Leserin und kein Leser auf die Idee kommen würde, die inszenierende Schreibweise lege eine fiktionale Darstellung nahe, im Gegenteil: Die nicht immer, aber doch regelmäßig auch durch das Präsens re-inszenierte Anwesenheit des Reporters vor Ort, seine Augenzeugenschaft, gilt im Rahmen der journalistischen Kommunikation als Authentifizierungstechnik.

Nun sind die journalistischen Textsorten und damit die entsprechenden Rezeptionskonventionen um 1850, als Fontanes Gedicht erscheint, noch nicht auf eine Weise etabliert, wie dies im zwanzigsten Jahrhundert der Fall ist. Doch auch um 1850 ist die simultane Darstellung eine journalistische Lizenz. Anders gesagt: Im historischen Rezeptionskontext ist die Darstellungsweise kein Signal für Fiktionalität.

Diese These ist zu belegen. Knapp anderthalb Monate vor Fontanes Gedicht, am 21. März 1850, erscheint im Morgenblatt ein Bericht mit dem Titel »Die Flüchtlinge in London«. Der Text beginnt folgendermaßen:

Märznebel umdüstern die große Stadt, was man hier Himmel nennt, ist eine graue Decke, die über den Dächern liegt und zum Zerschneiden einladet; ein Meteor, eine kleine runde Feuerkugel ohne Strahlen, in diese dichte Masse wie eingeschoben, heißt hier Sonne. ([anonym] 1850b, 269)

Die simultane Darstellungsweise wird auch nach dem atmosphärischen Einstieg fortgesetzt:

Dort in der engen Straße in einem Zimmer des dritten Stockwerks sitzen acht italienische Flüchtlinge beisammen. Sie haben die Wohnung seit einer Woche nicht mehr verlassen, weil sie ihre Röcke versezt, um Brod dafür zu kaufen. Das Feuer in ihrem Kamin ist längst erloschen, ihre Tage sind kurz, denn es fehlt ihnen die Leuchte, sie auszudehnen, und ihre langen Nächte verlängern sich durch die Schlaflosigkeit des Hungers. ([anonym] 1850b, 269)

Nichts am detaillierten Bericht, der im Monatsregister des Morgenblatts zu den »Aufsätzen vermischten Inhalts« gezählt wird, ${ }^{21}$ deutet darauf hin, dass hier eine

20 Wobei vor allem zu bemerken ist, dass Hempfer (2014) meines Erachtens zu schnell von >Inszenierung`auf `Fiktionalität` schließt. Überzeugender sind aus meiner Sicht Zymners Überlegungen zur Textfiguration, in denen es heißt: »Für den Fall aber, daß man es mit der Gestaltung einer Textfiguration des Autors zu tun bekommt, muß dies nicht von vornherein gegen die Faktualität des `Gesagten` und die Authentizität des `Sagens` sprechen« (Zymner 2009, 19).

21 Das Monatsregister ist im Halbjahrsband zur ersten Hälfte von 1850 (Morgenblatt 1850) nach Seite 308 eingebunden. 
Rezeption gemäß der Institution `Fiktionalitätı angebracht wäre. Hier schreibt jemand, journalistisch, von der Realität. Gleiches gilt für Reiseberichte dieser Zeit, die ebenfalls mit der Technik der simultanen Darstellung arbeiten. Exemplarisch sei auf einen Bericht über Zürich aus den Unterhaltungen am häuslichen Herd verwiesen. ${ }^{22}$

Eine derartige simultane Darstellung ist zwar im journalistischen Kontext, soweit ich sehe, nicht die Regel, zahlreiche Berichte arbeiten mit späterer Darstellung. Aber sie ist in faktualen Texten möglich und insofern indifferent hinsichtlich der Differenz >fiktional vs. faktualı. Die Simultaneität der Darstellung in Fontanes Gedicht mag also als eine Technik der >Inszenierung` wahrgenommen worden sein, ein eindeutiges Fiktionssignal ist sie nicht.

Eine solche Indifferenz bestimmter, häufig als starke Fiktionssignale gedeuteter Darstellungstechniken betrifft auch weitere Textphänomene - jedenfalls im Kontext der Zeitschriften der Jahrhundertmitte, für die verschiedentlich die »Unmöglichkeit der Trennung von Wahrheit, Faktualität, Bericht auf der einen und bloßer Wahrscheinlichkeit bzw. Fiktion auf der anderen Seite« (Günter 2008, 150) als charakteristisch benannt wurde. ${ }^{23}$ Vor diesem Hintergrund verliert auch ein zweites Textphänomen aus Fontanes Gedicht, das aus heutiger Sicht als dezidiert fiktionale Technik gilt, im historischen Rezeptionskontext seinen Signalcharakter.

Gemeint sind jene Passagen, in denen das Gedicht mit »Einblick[en] in die Psyche Dritter« arbeitet, nach Zipfel das »am meisten besprochene Fiktionssignal« (Zipfel 2014, 112). In Ein Ball in Paris werden solche Einblicke im Zusammenhang mit dem Kaiserneffen, also mit Louis Napoléon, gewährt, der in zwei längeren Passagen aus der Menge der Ballbesucher herausgehoben wird, wobei punktuell auch Aussagen über innere Vorgänge getätigt werden (unter anderem »Der Kaiserneffe aber - klanglos hin / Zieht heut der Töne Macht an seinem Sinn «; »Du Kaiserneffe, der im Herzen still / Noch immer rechnet, ob's nicht werden will? «; Fontane 1850, [485] und 486).

Auch solche Darstellungstechniken sind um 1850, wenn sie nebenbei erfolgen, innerhalb faktualer Texte lizenziert. Ein längerer, im Grundduktus unterhaltend-berichtender Artikel aus den Unterhaltungen am häuslichen Herd etwa, der vom »Leben der Vornehmen in England« handelt, erzählt auf vergleichbare

22 »Wir gehen weiter, oben jene stattliche Gebäudereihe entlang und treten in den Garten des St.=Leonhard=Pfründnerhauses. Welch ein ganz anderes Bild! Nur ein Stückchen vom See glänzt herüber [...] (Lampert 1859, 141).

23 Auch Helmstetter konstatiert, dass »die kategoriale Grenze zwischen Wirklichkeit und Fiktion - oder besser: zwischen journalistischem und literarischem Diskurs« in den Zeitschriften dieser Zeit »verwischt« werde (Helmstetter 1997, 254). 
Weise wiederholt aus einer nullfokalisierten bzw. multiperspektivischen Position heraus, zum Beispiel in der folgenden Passage, in der die Technik des Gedankenberichts angewendet wird (auch hier, nebenbei, in simultaner Darstellungsweise):

Ein anderes Kammermädchen tritt, nachdem sie angeklopft hat, ins Zimmer und meldet, daß Myladys Wagen vorgefahren ist.

»Er kann warten!« ruft Mylady. »Mary, helfen Sie Elisen nähen!«

Und die beiden Kammerjungfern bringen mit fliegenden Händen den Anzug zu Stande.

Elise denkt dabei an den Brief, den sie heute von einer Landsmännin und früheren Schulfreundin empfangen. [...] Elise überlegt, ob sie dem Beispiel der Freundin nicht folgen solle [...]. ([anonym] 1859, 139; meine Hervorhebungen, P. T.)

Bei Fontanes Gedicht kommt hinzu, dass sich im Laufe des Textes explizit ein deutendes und (auch im journalistischen Sinn) kommentierendes \Ich $>$ zu erkennen gibt, wodurch die Aussagen über die Vorgänge im Inneren des Kaiserneffen wie psychologisierende Zuschreibungen dieses `Ich deutenden und kommentierenden Perspektivträger lassen sich darüber hinaus die inszenierten »Schattenbilder « (Fontane 1850, 486) legitimieren, mit denen das `Ich`Szenen aus der Revolutionszeit imaginiert.

Sowohl die simultane Darstellung als auch die punktuelle Innensicht können, folgt man dieser Argumentation, innerhalb des historischen Rezeptionskontextes nicht als klare Fiktionssignale gewertet werden. Sie erweisen sich als indifferent, was den Status der Texte betrifft. Nicht berücksichtigt wurde bisher allerdings, dass es sich bei »Ein Ball in Paris « um ein `Gedicht handelt, verfasst in Versform, wohingegen die zur Relativierung des fiktionsindizierenden Charakters angeführten Beispiele in Prosa verfasst sind. Nun ist die Gedichtform, wie Zipfel (2001, 303-304) argumentiert hat, aus systematischer Sicht weder ein Hinweis auf Faktualität noch auf Fiktionalität. Diese systematische Aussage schließt jedoch nicht aus, dass die generische Form historisch mit spezifischen Rezeptionskonventionen verknüpft sein könnte. Es ist in diesem Sinne zu diskutieren, welchen Status `Gedichte im historischen Rezeptionskontext aufweisen.

\subsection{Das `Gedicht ` im Morgenblatt um 1850 und in zeitgenössischen Poetiken}

Um zunächst die für das Morgenblatt charakteristische Idee vom `Gedicht` zu ermitteln, bietet es sich an, die redaktionellen Praktiken der generischen Einordnung zu untersuchen. Hier fällt als erstes auf, dass in den Ausgaben des Morgenblatts keine stabile Rubriken-Ordnung besteht. Allein die »Korrespondenz-Nachrichten« sind 
als Rubrik fest etabliert und werden entsprechend stets, und jeweils am Ende der Ausgabe, durch eine eigene Überschrift angezeigt. Die anderen, vorderen Bereiche der Hefte versammeln hingegen ohne festes Schema unterschiedliche Textsorten und Genres. Eine generische Rubrizierung erfolgt dann erst in den Monatsregistern, die in dem mir vorliegenden Halbjahresband (siehe Morgenblatt 1850) jeweils nach der letzten Ausgabe eines Monats eingebunden sind. Neben den »Korrespondenz-Nachrichten « werden hier drei weitere Rubriken aufgeführt: »Gedichte«, »Erzählungen«, »Aufsätze vermischten Inhalts«.

Keine dieser Klassifikationen ist besonders einschlägig in jenen Systematiken um 1850, die - in populär konventionalisierter Form - etwa in den zahlreichen Poetiken und Rhetoriken oder Anthologie und Musterbüchern dieser Zeit verhandelt werden. Am deutlichsten bestimmt sind wohl noch die »Aufsätze«, die beispielsweise im Lehrbuch des deutschen Prosastiles von Friedrich Beck aus dem Jahr 1861 als Form der »reinen Kunstprosa« klassifiziert werden: In dieser Form werde ein »historisches oder rationales Thema in Kürze behandelt» (Beck 1861, 67). Insbesondere für den schulischen Gebrauch gibt es zudem diverse Mustersammlungen mit Aufsätzen, unter anderem von Hermann Kletke (1844). In jedem Fall ist in diesem Zusammenhang eindeutig, dass es sich bei einem `Aufsatz « um eine faktuale Textsorte handelt.

Keineswegs so eindeutig ist der Status hingegen im Fall der »Erzählungen«, die in zeitgenössischen Poetiken in der Regel von den >Poetischen Erzählungen unterschieden werden, wobei vor allem. das Verskriterium den Unterschied macht. Dabei stellt die >Erzählung`, so Knüttell 1848 in seinem Lehrbuch Die Dichtkunst und ihre Gattungen, »in ungebundener Rede eine interessante Begebenheit dar, sei diese nun wirklich vorgefallen, oder auf eine den Erscheinungen des wirklichen Lebens entsprechende Weise erfunden« (Knüttell 1848, 151). Diese Status-Indifferenz zeigt sich auch bei den `Erzählungen` im Morgenblatt, die auf eine für die Zeitschriftenliteratur in der Mitte des neunzehnten Jahrhunderts typische Weise mit nur sehr schwachen Statussignalen ausgestattet sind. In vielen Fällen lässt sich am Text selbst erst aus einer dezidiert analytischen, teils recherche-gestützten Perspektive entscheiden, ob es sich um eine faktuale oder eine fiktionale >Erzählung handelt.

In diesem Umfeld der Statusunbestimmtheit stehen nun auch die Texte der Rubrik »Gedichte« des Morgenblatts. Prinzipiell folgt die Kategorisierung von Texten als >Gedicht $\iota$ dabei der allgemeinen Schematik der Poetiken, nach denen auch um 1850 zumeist noch all das als >Gedicht` gelten kann, was in Versen verfasst ist - sei es nun ein episches, ein lyrisches, ein dramatisches, oder gelegentlich auch noch ein didaktisches Gedicht. Zugleich sind die im Morgenblatt als >Gedicht ‘ abgedruckten Texte in der Regel dem am ähnlichsten, was in den Poetiken unter der Bezeichnung ılyrische Dichtung` diskutiert wird; Dramen gibt es 
im Morgenblatt dieser Zeit keine, und nur einzelne `Gedichte stehen dem (dann allerdings sehr kurzen) $>$ Epos` nahe.

Die Rubrizierung im Morgenblatt liefert also, jedenfalls vor dem Hintergrund der generischen Ordnungen der Zeit, keine klaren Hinweise auf stabile Zuordnungen und in Folge dessen greifende Konventionen des Umgangs mit den einzelnen Exemplaren. In der Rubrik »Gedichte« begegnen denn auch sehr unterschiedliche Texte, etwa liebesliedhafte Formen von Justinus Kerner (1850) oder politische Kommentare zum Zeitgeschehen, wie »Deutschland an Straßburg« vom journalistisch umtriebigen Hermann Marggraff.

Die Ketten, die mit Frankreich dich verschlungen,

Du nennst sie sanft und zart gleich seidnen Schnüren (Mar[g]graff 1850, 566). ${ }^{24}$

Hinzu kommen im Kern deskriptiv angelegte Texte, wie das antikisierende »Aus der Glyptothek«.

Seh' ich die jonischen hier, dort schlanke korinthische Säulen,

Auf dem gebreiteten Grund mächtiger Stufen erhöht ([anonym] 1850a, [13])

Dabei zeigen gerade die politisch kommentierenden und die deskriptiven Verstexte, dass die Morgenblatt-Rubrik »Gedichte« selbstverständlich auch die Möglichkeit faktualen ১Sprechens Paris « kann insofern im historischen Rezeptionskontext nicht als Signal für eine bestimmte Statuszuschreibung gelten.

Dennoch ist die Versform nicht unerheblich. Zwar markiert sie keinen spezifischen Status der 〉Rede` bzw. des `Sprechers`, sie muss jedoch im Kontext des Morgenblatts insgesamt als eine seltene Form gewertet werden. In den 155 Ausgaben des ersten Halbjahresbandes 1850 erscheinen bloß 16 Gedichte; einige davon erstrecken sich über mehrere Ausgaben, sodass in insgesamt 20 Ausgaben Gedichte abgedruckt sind, also in etwas mehr als 12\% aller Ausgaben. Zählt man lediglich die Seiten, auf denen Gedichte erscheinen, dann zeigt sich ein noch deutlicheres Bild der Seltenheit der als `Gedicht` rubrizierten Form: Nur auf 38 der 620 Seiten des ersten Halbjahresbandes, also auf knapp 6\% der Seiten, finden sich Gedichtverse.

Es ist davon auszugehen, dass diese Seltenheit der Form mit einer, und sei es diffusen, Semantik einhergeht, dass also allein das von der Versform geprägte

24 Das Gedicht erschien mit dem in Klammern gesetzten, in Form eines Untertitels gestalteten Zusatz: »Bei der Nachricht von der beschlossenen Errichtung eines Denkmals zum Gedächtniß der Einverleibung in Frankreich.» 
Layout eines Gedichts bestimmte Vorstellungen und Erwartungen hervorrief. Einen Hinweis auf diese Semantik des Gedichts im Morgenblatt um 1850 liefert die Neujahrsausgabe.

$\mathrm{Zu}$ Beginn dieser Ausgabe bringt das Morgenblatt einen prosaischen Text der Redaktion (bzw. des Redakteurs Hermann Hauff) »Zum Antritt des Jahres 1850«, der zum erheblichen Teil aus einer Begründung dafür besteht, dass die Neujahrsausgabe nicht, wie gewohnt, von einem Gedicht eröffnet wird. Da dieser programmatische Text so etwas wie das implizite generische Konzept des Gedichtes im Morgenblatt dieser Zeit verhandelt, sei der entsprechende Textteil - es ist der Anfang - etwas länger zitiert:

Bisher trat höchst selten der Jahreswechsel ein, ohne daß er den Lesern dieser Blätter irgend einen wohlgemeinten poetischen Neujahrsgruß und Wunsch gebracht hätte. Fast immer war freiwillig oder aufgefordert einer unserer dichterischen Freunde zur Hand, der in den ersten Spalten des frischen Jahrgangs auf seiner Leier ein Stück spielte, in dem sich an die heitere oder ernste Musterung des abgelaufenen das Horoskop des angetretenen Jahres knüpfte. Wenn wir dießmal dieser Sitte untreu werden, so rechnen wir nicht allein auf die Nachsicht, sondern fast auf den Dank der Leser.

Es ist wohl keiner, der nicht in diesen lezten Stunden eines bedeutungsschweren Jahres mit doppeltem Ernste die Summe unseres öffentlichen Jammers und die Wechselfälle des eigenen kleinen Schicksals überrechnete. Da müßte es ihn fast widrig berühren, wenn ihm einer am Neujahrstag das Elend, das er mit angesehen und selbst erlebt, in schweren Spondäen noch einmal vorwöge, oder in tanzenden Daktylen es ihm auszureden suchte. [...] Armer Poet, der mit der längst entbehrten, neuerdings kaum vermißten Gabe des wahrhaft Schönen sich zu einer Zeit an die Straße sezte, wo Alles mit zerstreuten Blicken daran vorüber eilte! Unsere Besorgniß war ungegründet; dagegen wäre es uns nicht schwer geworden, einen gebildeten, in einer der gangbaren Stylarten wohlgeübten Leierspieler aufzutreiben, der seine lyrische Walze auf Uhlands sinnig gravitätische Tonweise gestellt hätte, oder aber auf die pathetisch gezwungene Freiligraths, oder auf die bittersüß spöttische Heines. Jedenfalls wären wir im Stande gewesen, den Lesern ein braves Gedicht zuzusichern, wenn nur nicht zu allen Zeiten mit den braven Gedichten so wenig herausgekommen wäre, als in den lezten Jahren in unserer Oeffentlichkeit mit den bloß braven, selbst edeln Männer. Der Poet, dem wir das Wort gegeben hätten, wollte er gut brüllen, mußte schaudernd die mit Blut und Thränen beschriebenen Blätter unserer nächsten Vergangenheit vor uns aufrollen und aus der geballten Wortwolke Blitze zucken lassen, die Alles niederschmetterten, was der deutschen Freiheit und Einheit hemmend entgegentritt [...]. (H[auff] 1850, [1])

Die Gedichtform wird von Hauff als eine politische verhandelt. Das Gedicht als literarisches, vor allem aber als publizistisches Format wird mit der politischen Situation Neujahr 1850 gekoppelt. Verworfen werden im Zuge dessen diverse Optionen der öffentlichen Rede in Gedichtform, wobei diese summarisch unter der Bezeichnung »brave[ ] Gedichte[ ] zusammengefasst sind. Angesichts der aktuellen, postrevolutionären Situation wird diesen `braven Gedichten ‘ die Wirkmacht abgesprochen. Ihnen entgegengestellt wird ein Typus des Gedichts, der - 
die «nächste[ ] Vergangenheit« thematisierend - mit wütendem Engagement (»brüllen«, »aus der geballten Wortwolke Blitze zucken lassen«, »Alles niederschmetter[n]«) politisch Position bezieht.

Diese politische Semantisierung der Form 〉Gedicht` lässt sich als Teil des historischen Rezeptionskontextes begreifen. Ein Gedicht, das in den folgenden Ausgaben des Morgenblattes erscheint, muss die von der Redaktion artikulierten, hehren Erwartungen zwar nicht notwendig erfüllen; doch diese stehen als Maßstab im Raum, zumal bei einem Gedicht, das sich, wie »Ein Ball in Paris«, der »nächsten Vergangenheit« annimmt. Insofern liegt es nahe, dass Fontanes Gedicht als eine Form des - redaktionell akzeptierten - politischen Sprechens eines engagierten Subjekts wahrgenommen wurde. Für die spätere, literaturwissenschaftliche Rezeption ist eine solche Einordnung auch belegt: Das >Ich in Fontanes Gedicht erfülle »die Aufgabe einer kritischen Sonde zur Erkenntnis der Gesellschaft um 1850«, es erweise sich als »kritisch, mahnend«, als »zeitgeschichtliche[s] Gewissen gleichsam« (Hasubek 2001, 61), urteilt Peter Hasubek in seiner Interpretation, und das sogar, ohne dass der entschieden politisierte Gedichtbegriff des Morgenblatts (das als Publikationsort nicht berücksichtigt wird) dabei eine Rolle spielt.

Im historischen Rezeptionskontext des Erscheinens im Morgenblatt 1850 liefert die Gedichtform von »Ein Ball in Paris« keine grundsätzlichen Hinweise auf einen spezifischen Status des Adressanten. Wo sie als seltene Form überhaupt Verwendung findet, wird sie vielmehr im Kontext der Forderung nach einem politischen `Sprechen im Gedicht wahrgenommen. Vom Adressanten eines solchen Gedichtes war die politische Stellungnahme gefragt. Das führt nicht notwendig dazu, dass der historische Leser von »Ein Ball in Paris« den Adressanten mit dem Autor Fontane identifiziert. Es ist aber davon auszugehen, dass Gedichte mit politischen Themen in dieser historischen Situation und in diesem publizistischen Umfeld als authentische Kommentare zur Gegenwart begriffen wurden. Selbst wenn sie gewissermaßen journalistisch sinszeniert « waren.

Dieses Argument lässt sich noch einmal schärfen, wenn man einbezieht, welches allgemeine Verständnis vom Adressanten eines lyrischen Gedichts in den Diskussionen um 1850 vertreten wurde. Denn tatsächlich gehen die - späterhin vielgeschmähten - populären Definitionen der lyrischen Dichtung im neunzehnten Jahrhundert nahezu stets davon aus, dass die Lyrik jene Dichtungsart

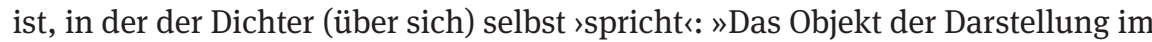
lyrischen Gedichte sind die selbsteigenen Gefühle des Dichters « (Dieckhoff 1832, 181) ist im Handbuch der Poetik für Gymnasien von 1832 zu lesen; im Lehrbuch der Poetik heißt es drei Jahre später: „Der Lyriker spricht sein Gefühl, sein Inneres aus« (Uschold 1835, 46); »Alle lyrischen Dichtungsarten kommen darin überein, daß sie der Ausdruck eines selbstständigen Gefühls des Dichters sind « (Pablasek 
1848, 16), definiert 1848 die Deutsche Poetik; der lyrische Dichter stelle »seine Person, sich als Subjekt, in den Vordergrund«, bestimmt 1860 das Lehrbuch der Poetik (Koepert 1860, 118).

Dabei wird in der Regel durchaus die Möglichkeit erwogen, dass der Dichter nicht selbst ıspricht`, sondern eine `Sprecher«-Figur erfindet, dass es also (um die oben vorgeschlagene, an Zymner angelehnte Unterscheidung aufzugreifen) auktoriale und personale Gedichte gibt. ${ }^{25}$ Diese Option eines erfundenen GedichtAdressanten, einer lyrischen Persona, ist aber in den Poetiken stets eine nachgeordnete. Als default-Modus des lyrischen Gedichts gilt, dass darin der Dichter selbst `spricht ` - primär über seine eigenen `Gefühle`, in anderer Variante auch über seine Gedanken, Ansichten, Wahrnehmungen, seinen Blick auf die Welt: wie Fontane in »Ein Ball in Paris«.

\section{Resümee}

Die zurückliegenden Ausführungen haben eine Reihe von Argumenten zusammengetragen, die es meines Erachtens sinnvoll erscheinen lassen, dass im historischen Rezeptionskontext, der mit dem Zeitschriftenabdruck von »Ein Ball in Paris« gegeben war, eine zeitgenössische Leserin, ein Leser davon ausging, dass Fontane der `Sprecher`, der Adressant des Gedichtes ist. Die Anwesenheit des Autors vor Ort musste als möglich gelten, potenzielle Fiktionssignale hatten im Zeitschriftenkontext keine Signalfunktion, der Leser war durch die Publikationspolitik der Morgenblatt-Redaktion eingestimmt auf Gedichte mit politischen Stellungnahmen, wobei ihm lyrische Gedichte, im Sinne der poetologischen Diskussion dieser Zeit, grundsätzlich als `Rede` ihres Autors galten. - Vor diesem Hintergrund, angesichts dieses historischen Rezeptionskontextes, halte ich es für unwahrscheinlich, dass eine Leserin bei der Lektüre eine zusätzliche Kommunikationsinstanz in Form eines fingierten Adressanten ansetzt. Autor und Adressant des Gedichts im Morgenblatt war »Th. Fontane«, so die Angabe am Ende des Abdrucks.

Der zurückliegende Versuch, am Beispiel von Fontanes Gedicht die Wahrscheinlichkeit historischer Statuszuschreibungen zu rekonstruieren, basiert auf einem Verständnis von »Ein Ball in Paris« als journalistischem Gedicht mit einem gerade nicht biographisch konturierten `Sprecher`, sondern einem >publizisti-

25 Vgl. z. B.: „Sodann giebt es auch lyrische Gedichte, in welchen der Dichter nicht seine eigenen Gefühle schildert, sondern eine andere, entweder wirkliche oder fingirte Person, in deren Geist und Lage er sich hineindenkt, Gefühle aussprechen läßt« (Kleinpaul 1856, 137). 
schen Subjekt als Kommunikationsinstanz, als Adressant. Nicht nur in der lyrikologischen Diskussion des zwanzigsten und einundzwanzigsten Jahrhunderts, auch in den Poetiken des neunzehnten Jahrhunderts werden solche Gedichte in der Regel nur nebenbei thematisiert. In dieser Hinsicht gilt, auch über das neunzehnte Jahrhundert hinaus, was Robert Prutz 1845 in seiner Schrift über Die politische Poesie der Deutschen festgestellt hat, dass nämlich die politische Dichtung von den >Ästhetikern` weitgehend ignoriert wird:

Denn nach der Meinung dieser Partei ist die Dichtung göttlicher Natur, ihr Reich ist nicht von dieser Welt, sie ist der Friede, die Harmonie, der selig unbefangene Genuß des Schönen; was hätte sie also zu theilen mit den irdisch vergänglichen, den feindseligen und häßlichen Erscheinungen der Tagesgeschichte? (Prutz 1845, 254)

Eingreifende, die `Tagesgeschichte darstellende und kommentierende Gedichte hatten in den Poetiken keinen Ort. Allein in der meist an den Rand des künstlerischen Ausdrucks gedrängten Kategorie des >Gelegenheitsgedichts ${ }^{{ }^{26}}$ war Platz für sie. Insofern verwundert es nicht, dass Ein Ball in Paris, das Fontane in der ersten Auflage seiner Gedichte noch ohne jede Rubrizierung abdrucken ließ, in späteren Auflagen unter die »Gelegenheits-Gedichte« geordnet wurde. Dass Fontane es zudem, wie einleitend erwähnt, um die Angabe »(Dezember 1849)« ergänzt und damit die konkrete, zeitgeschichtliche Referenz markiert, fügt sich in diese Deutung von Ein Ball in Paris als journalistisches Gelegenheitsgedicht, das - gemäß der Logik des Journalismus, in dem sich Fontane gerade zu etablieren versucht - ohne die historische Gelegenheit des Pariser Balls, ohne das Bedürfnis nach und die publizistische Möglichkeit des Autors zur politischen Stellungnahme sowie ohne die redaktionell proklamierte Politisierung der Gedichtform sicher nicht entstanden, nicht publik geworden wäre.

Selbst wenn die im Zurückliegenden entwickelte Argumentation überzeugend dargelegt haben sollte, dass die Aussage »Fontane ist der Adressant von Ein Ball in Paris « in einer gewissen, die historische Situation rekonstruierenden Hinsicht Sinn ergibt, ist abschließend noch einmal die Aufwendigkeit und Interpretationsabhängigkeit der Argumentation hervorzuheben. Beides halte ich keineswegs für

$26 »[$ W] enn irgend ein Vorfall zur bloßen Veranlassung für den Dichter wird, sich darin oder darüber zu äußern«, läge ein Gelegenheitsgedicht vor, so beispielsweise Günther $(1845,38)$; wobei zugleich gilt: »die wenigsten Gedichte aber, welche einer bestimmten Gelegenheit ihre Entstehung danken, haben zugleich dichterischen Werth « (Buchner 1863, 58). 
zufällig, also nicht allein dem konkreten Fall dieses Fontane-Gedichts geschuldet. Im Gegenteil, mir scheint (und dies wäre in weiteren Studien zu prüfen), dass es für die Lyrik insgesamt weder charakteristisch ist, dass sie sauktorialı, noch, dass sie > personal vermittelt wird. Die lange Geschichte der lyrikologischen Diskussion über den `Sprecher` des Gedichts legt vielmehr die Vermutung nahe, dass konstitutiv für den `Sprecher eines Gedichts dessen Unter-, wenn nicht Unbestimmtheit sein könnte.

Am Ende hätte dann vielleicht doch Käte Hamburger Recht, die in der Logik der Dichtung auf die »Variabilität und Unbestimmtheit« des `Ichs` im Gedicht als »ein weiteres die lyrische von der nicht-lyrischen Aussage unterscheidendes Strukturkriterium« hingewiesen hat, wobei »zu dem Unbestimmtheitscharakter auch die Differenz oder Identität zwischen dem lyrischen Ich und dem DichterIch gehört« (Hamburger 1977, 249). Gerade für eine eigenständige Lyrikologie, die sich auch von der narratologischen Lyriktheorie emanzipieren will, könnte dieses Theorem von der prototypischen Unbestimmtheit der lyrischen >Stimme von Bedeutung werden.

\section{Literaturverzeichnis}

[anonym]. »Aus der Glyptothek«. Morgenblatt für gebildete Leser 44.4 (4. Januar 1850a): [13]-15. [anonym]. »Flüchtlinge in London« [1. Teil]. Morgenblatt für gebildete Leser 44.68 (21. März 1850b): 269-270.

[anonym]. »Das Leben der Vornehmen in England« [1. Teil]. Unterhaltungen am häuslichen Herd [N. F.] 5.9 (1859): 136-140.

Beck, Friedrich. Lehrbuch des deutschen Prosastiles für höhere Unterrichts-Anstalten, wie auch zum Privatgebrauche. München: E. A. Fleischmann, 1861.

Bers, Anna und Peer Trilcke. "Lyrik und Phänomene des Performativen. Problemaufriss, theoretische Perspektiven und Vorschläge zu einer künftigen Terminologie«. Phänomene des Performativen in der Lyrik. Systematische Entwürfe und historische Fallbeispiele. Hg. Anna Bers und Peer Trilcke. Göttingen: Wallstein, 2017. 9-58.

Berbig, Roland. Theodor Fontane Chronik. 5 Bde. Projektmitarbeit 1999-2004: Josefine Kitzbichler. Berlin: de Gruyter, 2010.

Berbig, Roland. Theodor Fontane im literarischen Leben. Zeitungen und Zeitschriften, Verlage und Vereine. Mitarbeit: Bettina Hartz. Berlin: de Gruyter, 2000.

Borkowski, Jan und Simone Winko: »Wer spricht das Gedicht? Noch einmal zum Begriff slyrisches Ich und zu seinen Ersetzungsvorschlägen «. Lyrische Narrationen - narrative Lyrik. Gattungsinterferenzen in der mittelalterlichen Literatur. Hg. Hartmut Bleumer und Caroline Emmelius. Berlin: de Gruyter, 2011. 43-77.

Brecht, Bertolt. »Aus allem etwas machen«. Sinn und Form 1.5 (1949): 103.

Brecht, Bertolt. »Kurzer Bericht über 400 (vierhundert) junge Lyriker«. Große kommentierte Berliner und Frankfurter Ausgabe. 21. Bd. Schriften 1: Schriften 1914-1933. Bearb. Werner Hecht. Berlin, Weimar: Aufbau und Suhrkamp, 1992. 191-193. 
Brecht, Bertolt. Große kommentierte Berliner und Frankfurter Ausgabe. 15. Bd. Gedichte 5: Gedichte und Gedichtfragmente. 1940-1956. Bearb. Jan Knopf und Brigitte Bergheim. Berlin: Aufbau und Suhrkamp, 1993.

Brecht, Bertolt. Große kommentierte Berliner und Frankfurter Ausgabe. 29. Bd. Briefe 2. Bearb. Günter Glaeser. Berlin: Aufbau und Suhrkamp, 1998.

Buchner, Wilhelm. Deutsche Dichtung. Die Lehre von den Formen und Gattungen derselben. Ein Leitfaden für Realschulen, höhere Bürger- und Töchterschulen. Essen: G. D. Bädeker, 1863.

Burdorf, Dieter. Einführung in die Gedichtanalyse. 3. Aufl. Stuttgart: Metzler, 2015.

Dg. [das heißt vermutlich Georg Bernhard Depping]. »[Korrespondenz-Nachrichten] Paris, December« [1. Teil]. Morgenblatt für gebildete Leser 44.1 (1. Januar 1850): 4.

Dieckhoff, Bernard. Handbuch der Poetik für Gymnasien. Münster: Theissingsche Buchhandlung, 1832.

Fischer, Carolin. Der poetische Pakt. Rolle und Funktion des poetischen Ich in der Liebeslyrik bei Ovid, Petrarca, Ronsard, Shakespeare und Baudelaire. Heidelberg: Winter, 2007.

Fontane, Th[eodor]. »Ein Ball in Paris«. Morgenblatt für gebildete Leser 44.122 (22. Mai 1850): [485]-486.

Fontane, Theodor: Gedichte [1. Aufl.]. Berlin: Carl Reimarus, 1851.

Fontane, Theodor: Gedichte. 2., vermehrte Aufl. Berlin: Wilhelm Hertz, 1875.

Fontane, Theodor: Große Brandenburger Ausgabe. [2. Abteilung] Gedichte. 1. Bd. Gedichte

(Sammlung 1898). Aus den Sammlungen ausgeschiedene Gedichte. Hg. Joachim Krueger und Anita Golz. 2. Aufl. Berlin: Aufbau, 1995.

Günter, Manuela. Im Vorhof der Kunst. Mediengeschichten der Literatur im 19. Jahrhundert. Bielefeld: transcript, 2008.

Günther, Friedrich Joachim. Die Poetik (Nach Hegel's Ästhetik). Für Gymnasien. Halle: Buchhandlung des Waisenhauses, 1845.

Hamburger, Käte. Die Logik der Dichtung. 3. Aufl. Stuttgart: Klett-Cotta, 1977.

H[auff], H[ermann]. »Zum Antritt des Jahres 1850« [1. Teil]. Morgenblatt für gebildete Leser 44.1 (1. Januar 1850): [1]-3.

Hasubek, Peter. »Ein Ball in Paris«. Gedichte von Theodor Fontane. Interpretationen. Hg. Helmut Scheuer. Stuttgart: Reclam, 2001. 49-64.

Helmstetter, Rudolf. Die Geburt des Realismus aus dem Dunst des Familienblattes. Fontane und die öffentlichkeitsgeschichtlichen Rahmenbedingungen des Poetischen Realismus. München: Fink, 1997.

Hempfer, Klaus W. Lyrik. Skizze einer systematischen Theorie. Stuttgart: Franz Steiner, 2014.

Kerner, Justinus. „Verse von Justinus Kerner«. Morgenblatt für gebildete Leser 44.39 (14. Februar 1850): [153].

Kleinpaul, Ernst. Die Lehre von den Formen und Gattungen der deutschen Dichtkunst. Für höhere Lehranstalten, sowie zum Selbstunterricht. 3. Aufl. Barmen: W. Langewiesche, 1856.

Kletke, Hermann (Hg.). Deutsche Aufsätze [... ] nebst Anmerkungen und Aufgaben. Für die oberen Classen höherer Bildungsanstalten wie auch zum Selbststudium. Berlin: Carl Friedrich Amelang, 1844.

Knopf, Jan. Bertolt Brecht. Stuttgart: Reclam, 2000.

Knüttell, August. Die Dichtkunst und ihre Gattungen. Ihrem Wesen nach dargestellt und durch eine nach den Dichtungsarten geordnete Mustersammlung erläutert. Mit Rücksicht auf den Gebrauch in Schulen. 2. Aufl. Breslau: Gratz, Barth und Comp., 1848.

Koepert, Hans Hugo Hermann. Lehrbuch der Poetik. Für Unterricht und Selbststudium. Leipzig: Arnoldische Buchhandlung, 1860. 
Köppe, Tilmann. »Die Institution Fiktionalität«. Fiktionalität. Ein interdisziplinäres Handbuch.

Hg. Tobias Klauk und Tilmann Köppe. Berlin: de Gruyter, 2014a. 35-49.

Köppe, Tilmann. »Fiktionalität in der Neuzeit«. Fiktionalität. Ein interdisziplinäres Handbuch.

Hg. Tobias Klauk und Tilmann Köppe. Berlin: de Gruyter, 2014b. 419-439.

Lampert, Friedrich. »Zürich«. Unterhaltungen am häuslichen Herd [N. F.] 5.9 (1859): 140-142.

Mar[g]graff, Hermann. »Deutschland an Straßburg». Morgenblatt für gebildete Leser 44.142

(14. 6. 1850): [565]-566.

Martínez, Matías. »Das lyrische Ich. Verteidigung eines umstrittenen Begriffs«. Autorschaft.

Positionen und Revisionen. Hg. Heinrich Detering. Stuttgart: Metzler, 2002. 376-389.

Mellmann, Katja und Marcus Willand. »Historische Rezeptionsanalyse. Zur Empirisierung von Textbedeutungen«. Empirie in der Literaturwissenschaft. Hg. Philip Ajouri, Katja Mellmann und Christoph Rauen. Münster: Mentis, 2013. 263-281.

Morgenblatt für gebildete Leser. [1. Halbjahr] 44 (1850). http://www.mdz-nbn-resolving.de/ urn/resolver.pl?urn=urn:nbn:de:bvb:12-bsb10531742-0. Digitalisat des MDZ München. (4. Oktober 2016).

Nürnberger, Helmuth. »Theodor Fontane: Leben und Persönlichkeit«. Fontane-Handbuch. Hg. Christian Grawe und Helmuth Nürnberger. Stuttgart: Alfred Kröner, 2000. 1-102.

Parker, Stephen R. und Matthew Philpotts. "Sinn und Form «. The Anatomy of a Literary Journal. Berlin: de Gruyter, 2009.

Pablasek, Matthias. Deutsche Poetik oder Lehre vom Silbenmaße, vom Versbau und von den Dichtungsarten. Wien: Friedrich Beck, 1848.

Petzold, Jochen. Sprechsituationen lyrischer Dichtung. Ein Beitrag zur Gattungstypologie. Würzburg: Königshausen \& Neumann, 2012.

Prutz, Robert Eduard. Die politische Poesie der Deutschen. (Aus Prutz's Taschenbuch). Leipzig: Otto Wigand, 1845.

Strobel, Jochen. Gedichtanalyse. Eine Einführung. Berlin: Erich Schmidt, 2015.

Uschold, Johann Nepomuk. Lehrbuch der Poetik. Für Gymnasien bearbeitet. Erster Theil. München: Joseph Lindauer, 1835.

Willand, Marcus. Lesermodelle und Lesertheorien. Historische und systematische Perspektiven. Berlin: de Gruyter, 2014.

Zipfel, Frank. Fiktion, Fiktivität, Fiktionalität. Analysen zur Fiktion in der Literatur und zum Fiktionsbegriff in der Literaturwissenschaft. Berlin: Erich Schmidt, 2001.

Zipfel, Frank. »Fiktionssignale«. Fiktionalität. Ein interdisziplinäres Handbuch. Hg. Tobias Klauk und Tilmann Köppe. Berlin: de Gruyter, 2014. 97-124.

Zymner, Rüdiger. Lyrik. Umriss und Begriff. Paderborn: Mentis, 2009.

Zymner, Rüdiger. »Begriffe der Lyrikologie«, im vorliegenden Band, 2019, S. 25-50. 